\title{
Calculation of structural-inhomogeneous multiply connected shell structures with viscoelastic elements
}

\author{
Tulkin Mavlanov* and Sherzod Khudainazarov \\ Department of Theoretical and Constructional Mechanics, Tashkent Institute of Irrigation and \\ Agricultural Mechanization Engineers, 39 KoriNiyoziy str., Tashkent 100000, Uzbekistan
}

\begin{abstract}
Using the basic relationships of the hereditary theory of viscoelasticity and asymptotic methods, the problem of natural oscillations of structural-inhomogeneous, multiply connected, axisymmetric shell structures is reduced to an effectively solvable mathematical problem of complex eigenvalues, in which approximate engineering methods are proposed.
\end{abstract}

\section{Introduction}

Algorithms for dynamic calculation of multiply connected structural-inhomogeneous shell structures have been developed within the framework of the V.V. Novozhilov linear theory of shells and the A.V. Aleksandrov physical relations, based on the A.V. Aleksandrov method of displacements and the S.K.Godunov method of orthogonal sweep. On the whole, the problem is reduced, using the basic relations of the hereditary theory of viscoelasticity and asymptotic methods, to an effectively solvable mathematical problem of complex eigenvalues in the framework of which the engineering methods are proposed.

To obtain the basic equations of the dynamics of structural-inhomogeneous multiply connected shell structures, the Lagrange variation equation is used:

$$
\sum_{p=1}^{N_{s}} \delta \ni_{p}+\sum_{i=1}^{N_{r}} \delta \ni_{i}+\sum_{e=1}^{N_{e}} \delta \ni_{e}-\sum_{p=1}^{N_{s}} \delta A_{p}-\sum_{i=1}^{N_{r}} \delta A_{i}=0
$$

where $\delta \ni_{p}$ is the variation of the potential strain energy of the p-th shell element; $\delta \ni_{\mathbf{e}}$-is the variation of the potential strain energy of the i-th ring element; $\delta \ni_{\mathbf{e}}-$ is the variation of the potential strain energy of the e-th viscoelastic connection; $\boldsymbol{\delta} \mathbf{A}_{\mathbf{p}}$ is the elementary work of external loads applied to the p-th shell element; $\boldsymbol{\delta} \mathbf{A}_{\mathbf{i}}$-is the elementary work of external loads applied to the $\mathrm{i}$-th ring element.

\footnotetext{
* Corresponding author: mavlanovt@inbox.ru
} 


\section{Method}

After some simple mathematical transformations, the task is reduced to solving the equations of the dynamics of shell structures with complex coefficients $[1,2]$ :

$$
\begin{gathered}
L_{p}+q_{p 0}+\omega_{R}^{2}\left[\bar{\rho}_{p}\right] U_{p}=0\left(\mathrm{p}=1,2, \ldots, N_{s}\right), \\
L_{r}^{i}+\left\|\theta_{i}\right\| f_{i 0}+\omega_{R}^{2}\left[G_{\omega}\right] \Delta_{i}+\sum_{j} \sum_{s}\left(\xi_{c i}^{i j s}\left[\bar{\eta}_{i}^{i j s}\right] Q_{i}^{i j s}+\sum_{j} \sum_{s}\left(\xi_{c i}^{i j s}\left[\bar{\eta}_{c i}^{i j s}\right] N_{c i}^{i j s}=0\right.\right. \\
\left(\mathrm{i}=1,2, \ldots, N_{r}\right)
\end{gathered}
$$

In the problem of natural oscillations of structures, the solution of equations (1), (2) is sought in the form

$$
U_{p}=U_{p} e^{-i \widetilde{\omega \tau}}, \quad \Delta_{i}=\Delta_{i} e^{-i \widetilde{\omega \tau}}
$$

Here $\widetilde{\boldsymbol{\omega}}-$ is the complex value of the oscillation frequency, the real part of which $\boldsymbol{\omega}_{\mathbf{R}}$ represents natural frequency, $\boldsymbol{\omega}_{\mathbf{I}}-$ is the damping coefficient. The equations of natural oscillations of structures have the form:

$$
\begin{gathered}
L_{p}+q_{p 0}+\widetilde{\omega}^{2}\left[\bar{\rho}_{p}\right] U_{p}=0 \quad\left(\mathrm{p}=1,2, \ldots, N_{s}\right) \\
L_{r}^{i}+\left\|\theta_{i}\right\| f_{i 0}+\widetilde{\omega}^{2}\left[G_{\omega}\right] \Delta_{i}+\sum_{j} \sum_{s}\left(\xi_{c i}^{i j s}\left[\bar{\eta}_{i}^{i j s}\right] Q_{i}^{i j s}+\right. \\
\sum_{j} \sum_{s}\left(\xi_{c i}^{i j s}\left[\bar{\eta}_{c i}^{i j s}\right] N_{c i}^{i j s}=0\right. \\
\left(\mathrm{i}=1,2, \ldots, N_{r}\right)
\end{gathered}
$$

The values of $\widetilde{\omega}^{*}$, for which there is a nontrivial solution of the system with complex coefficients (3) are the complex values of natural frequencies of the oscillations of the structural-inhomogeneous shell structures under consideration. Each of these equations describes the behavior of an individual shell element of a thin-walled shell structure. In our case, the difference from the known equations is of fundamental character, and the solution of these equations is complex due to the complexity of physical relationships that describe the structural inhomogeneity and rheological properties of the individual layers and the hereditary connections of the shell element. Each of these relationships is an equation of oscillations in the complex form of an individual stringer or bulkhead of the considered multiply connected structural-inhomogeneous shell structure, taking into account reactions from adjacent shell elements and viscoelastic connections. Vector $Q_{i}^{i j s}$ is, as seen from the expressions for $Q_{p}$ and $W_{p}$, the vector of generalized reactions from the ijs-th shell element adjacent to the $\mathrm{i}$-th ring or stringer element in the local coordinate system of the shell element under consideration. Vector $N_{c i}^{i j s}$ is, in turn, the vector of generalized reactions from the ijs-th and visco-elastic connection adjacent to the i-th ring or stringer element in the local coordinate system.

Matrices $\left[\bar{\eta}_{i}^{i j s}\right],\left[\bar{\eta}_{c i}^{i j s}\right]$ and coefficients $\xi_{i}^{i j s}, \xi_{c i}^{i j s}$ are the matrices and coefficients of transformation coefficients from the local coordinate system of the shell element or viscoelastic connection to the coordinate system of the shell structure under consideration.

In the case when there are no rod or ring elements in the node joint of two or more shell elements or viscoelastic connections, the first and third terms of the corresponding equation (2) are identically zero. This solution is interpreted as follows: the sum of the reactions of 
shell elements and viscoelastic connections converging at this node, plus the sum of external loads applied to the node in question, is zero.

Consider the solution of a number of practical problems of design of dynamic characteristics of a wide class of axisymmetric structural-inhomogeneous structures. To determine the frequencies and modes of natural oscillations, the problem-oriented procedure outlined above is used. It is known that the mathematical convergence of each of these methods has been widely and adequately studied. The question of the convergence of the proposed algorithm for the numerical implementation of problems of structuralinhomogeneous viscous-elastic shell structures as a whole has been studied insufficiently and presents an independent problem.

\section{Results}

An assessment of practical convergence of the algorithms is proposed, it is based on a comparison of the results found in literature to solve the test problems and the results of other solutions obtained in the framework of the developed methods, as well as with the results extended to complex arithmetic. As test examples, the shell structures have been chosen for which exact or approximate solutions are available.

1. To calculate the stiffness matrices of shell elements made of viscoelastic material, the orthogonal sweep method is used. Mathematical convergence and stability of this method as applied to the theory of elastic shells are widely investigated in publications of Ya.M. Grigorienko, V.I. Myachenkov, A.N. Frolov, V.P. V.P.Maltsev, T.Mavlanov and others.

Here, a study has been conducted of the convergence and stability of the orthogonal sweep method using complex arithmetic. Consider some of the results of this study for structural-inhomogeneous viscoelastic shell elements, whose rheological properties are described by the relaxation kernel $\mathrm{R}(\mathrm{t})=\mathrm{A} \exp (-\beta \mathrm{t}) t^{\alpha-1}$. It should be noted that, in contrast to [1], we took into account the effects of viscoelastic properties of structural elements.

As the first example of using the STIFMZ procedure, calculate the stiffness matrix of complex values for a four-layer rectangular plate (figure 1) with a different number of orthogonalization points $m$. The plate has the following geometrical and mechanical parameters: $\mathrm{a}=\mathrm{b}=100 \mathrm{~cm} ; \mathrm{j}=1,2, \quad 3, \quad 4 ; \quad \mathrm{h}_{\mathrm{j}}=0.25 \mathrm{~cm} ; E_{j}=2.10^{6} \mathrm{H} / \mathrm{cm}^{2} ; \quad \gamma_{j}=0.3 ; \rho_{j}=$ 7.8. $10^{-6} \mathrm{Hf} / \mathrm{cm}^{4} ; \mathrm{A}_{\mathrm{j}}=0.03 ; \beta_{j}=0.003 ; \alpha_{j}=0.1 ; \omega_{R}=300 \mathrm{~s}^{-1}$.

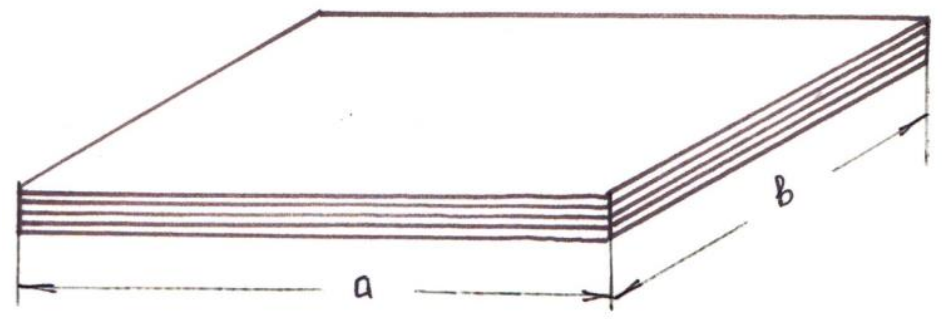

Fig.1. Four-layer rectangular plate.

The results of calculation for $\mathrm{m}=2,4,8,16,32,64$ and one integration step between points ( $m$ is the number of orthogonalization points on the interval of $a$ length) are presented in table 1. The top row of each element of the matrix represents the real component, and the bottom row -the imaginary component of this element.

The tables show that the practical convergence in calculating the matrix $[\mathrm{K}]$ is reached even at the number of orthogonalization points equal to $\mathrm{m}=4$, and at $\mathrm{m}=32$ and $\mathrm{m}=64$, 
the elements of the matrix $[\mathrm{K}]$ coincide in all significant digits. The symmetry of the matrix is achieved at $\mathrm{m}=16$.

As a second example, consider a three-layer cylindrical panel symmetrical along the thickness of the structure whose surface has the shape of a parabola (figure 2) and is determined by the equation

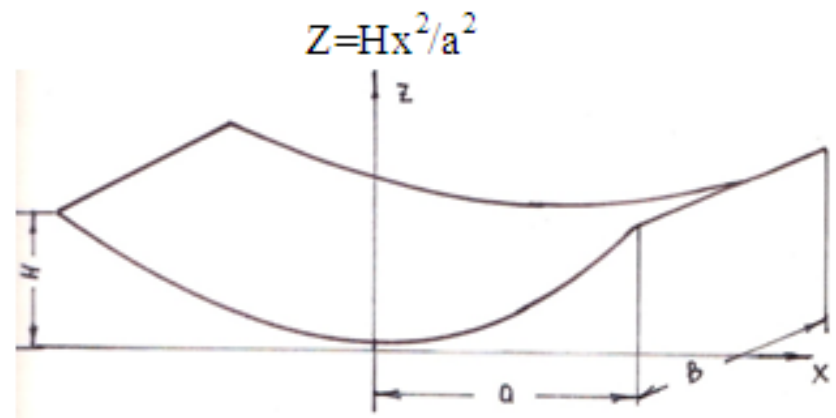

Fig.2. Three-layer cylindrical panel

The Lame coefficient $A_{1}$ and the curvature $k_{1}$ for this surface are calculated by the formulas

$$
\mathrm{A}_{1}=\sqrt{1+(H x / a)^{2}}, \mathrm{k}_{1}=-\left(1 / \mathrm{A}_{1}\right)^{3}\left(\mathrm{H} / \mathrm{a}^{2}\right)
$$

The panel has the following geometrical and mechanical characteristics: $\mathrm{a}=2500 \mathrm{~cm}$; $\mathrm{b}=5000 \mathrm{~cm} ; \mathrm{H}=250 \mathrm{~cm} ; \mathrm{h}_{1}=\mathrm{h}_{3}=1 \mathrm{~cm} ; \mathrm{h}_{2}=10 \mathrm{~cm} ; \mathrm{E}_{1}=\mathrm{E}_{3}=2.10^{7} \mathrm{H} / \mathrm{cm}^{2} ; \mathrm{E}_{2}=10^{6} \mathrm{H} / \mathrm{cm}^{2}$; $\gamma_{1}=\gamma_{3}=0.3 ; \quad \gamma_{2}=0.1 ; \quad \rho_{1}=s ; \rho_{3}=8.10^{-5} \quad \mathrm{Hfs}^{2} / \quad \mathrm{cm}^{4} ; \rho_{2}=2.10^{-5} \quad \mathrm{Hc}^{2} / \mathrm{cm}^{4}$; $\mathrm{A}_{1}=\mathrm{A}_{3}=\alpha_{1}=\alpha_{3}=\beta_{3}=0 ; \mathrm{A}_{2}=0.05 ; \alpha_{1}=0.1 ; \alpha_{2}=0.01 ; \beta_{3}=200 \mathrm{~s}^{-1}$.

The results of calculation for $\mathrm{m}=2,4,8,16,32,64$ and one integration step between points ( $\mathrm{m}$ is the number of orthogonalization points on the interval of $a$ length) are presented in table 1. The given examples show that using the S.K. Godunov orthogonal sweep method, it is possible to calculate with high accuracy the stiffness matrices of complex values.

2.1. To find the complex values of the natural oscillations frequencies of structuralinhomogeneous shell structures, i.e. when finding the roots of a nonlinear functional equation in complex variables

$$
\mathrm{D}(\widetilde{\omega)}=|\mathrm{P}(\widetilde{\omega})|=0
$$

the Muller method is used. The aspect of convergence of the iterative process proposed by Muller also requires research. This problem is practically solved in the following way. The algorithm for determining the frequencies and modes of oscillations of structuralinhomogeneous viscouselastic shell structures provides for the derivation of the protocol for finding the roots of equation (3), i.e. a computing engineer can always use this protocol to control the iterative process. Besides, one of the results of the algorithm is the relative accuracy in determining these roots achieved in the process of solving. Some data from these protocols are given in tables 1-2; the solution protocol contains the following data: the number of waves in the longitudinal direction $(\mathrm{N})$; the real $(\mathrm{QR})$ and imaginary $(\mathrm{QI})$ components of the complex frequency value $\widetilde{\omega}^{*}$; the real (DR) and imaginary (DI) components of the mantissa of the determinant $|P(\widetilde{\omega})|$; determinant order (IS) $|P(\widetilde{\omega})|$.

Final oscillation frequency values for the first example (table 1) are: $\omega_{R}^{*}=4.09759 ; \omega_{I}^{*}$ $=0.0748687$; relative accuracy $\varepsilon_{R}=3.54 .10^{-6} ; \varepsilon_{I}=6.8 .10^{-5}$. 
Table 1

\begin{tabular}{|c|c|c|c|c|c|}
\hline $\mathbf{N}$ & $\mathbf{Q R}$ & $\mathbf{Q I}$ & $\mathbf{D R}$ & $\mathbf{D I}$ & IS \\
\hline 1 & $5.4593 \mathrm{E}+00$ & $0.0000 \mathrm{E}+00$ & $9.7342 \mathrm{E}+00$ & $-2.0188 \mathrm{E}+00$ & 80 \\
\hline 1 & $5.4675 \mathrm{E}+00$ & $0.0000 \mathrm{E}+00$ & $9.9396 \mathrm{E}-01$ & $-2.0294 \mathrm{E}-01$ & 81 \\
\hline 1 & $5.4757 \mathrm{E}+00$ & $0.0000 \mathrm{E}+00$ & $1.0151 \mathrm{E}+00$ & $-2.0387 \mathrm{E}-01$ & 81 \\
\hline 1 & $5.1447 \mathrm{E}+00$ & $-3.0728 \mathrm{E}-01$ & $3.1727 \mathrm{E}+00$ & $-3.9077 \mathrm{E}+00$ & 80 \\
\hline 1 & $4.9792 \mathrm{E}+00$ & $-5.1567 \mathrm{E}-01$ & $1.0939 \mathrm{E}+00$ & $-3.6966 \mathrm{E}+00$ & 80 \\
\hline 1 & $4.7416 \mathrm{E}+00$ & $-8.4313 \mathrm{E}-01$ & $-7.6850 \mathrm{E}-01$ & $-2.8779 \mathrm{E}+00$ & 80 \\
\hline 1 & $4.4801 \mathrm{E}+00$ & $-1.3494 \mathrm{E}-01$ & $8.5936 \mathrm{E}-01$ & $-6.3399 \mathrm{E}-01$ & 80 \\
\hline 1 & $4.2435 \mathrm{E}+00$ & $-3.9976 \mathrm{E}-02$ & $3.5008 \mathrm{E}+00$ & $-6.7106 \mathrm{E}-01$ & 79 \\
\hline 1 & $4.0974 \mathrm{E}+00$ & $-5.0428 \mathrm{E}-02$ & $2.2221 \mathrm{E}+00$ & $4.8778 \mathrm{E}+00$ & 78 \\
\hline 1 & $4.0977 \mathrm{E}+00$ & $-7.5445 \mathrm{E}-02$ & $-3.9219 \mathrm{E}-01$ & $-1.2154 \mathrm{E}+00$ & 77 \\
\hline 1 & $4.0976 \mathrm{E}+00$ & $-7.4869 \mathrm{E}-02$ & $-6.1493 \mathrm{E}-01$ & $-5.9784 \mathrm{E}-01$ & 74 \\
\hline
\end{tabular}

Table 2

\begin{tabular}{|c|c|c|c|c|c|}
\hline $\mathbf{N}$ & $\mathbf{Q R}$ & $\mathbf{Q I}$ & $\mathbf{D R}$ & $\mathbf{D I}$ & IS \\
\hline 2 & $2.8474 \mathrm{E}+01$ & $0.0000 \mathrm{E}+00$ & $-7.0908 \mathrm{E}+00$ & $4.3724 \mathrm{E}-01$ & 93 \\
\hline 2 & $2.8517 \mathrm{E}+01$ & $0.0000 \mathrm{E}+00$ & $-7.1773 \mathrm{E}-00$ & $4.5420 \mathrm{E}-01$ & 93 \\
\hline 2 & $2.8560 \mathrm{E}+01$ & $0.0000 \mathrm{E}+00$ & $-7.2633 \mathrm{E}+00$ & $4.7109 \mathrm{E}-01$ & 93 \\
\hline 2 & $2.5678 \mathrm{E}+01$ & $-2.7600 \mathrm{E}-01$ & $-7.3030 \mathrm{E}+00$ & $-6.4527 \mathrm{E}-01$ & 91 \\
\hline 2 & $2.5654 \mathrm{E}+01$ & $-2.8160 \mathrm{E}-01$ & $-7.2172 \mathrm{E}+00$ & $-1.6035 \mathrm{E}+00$ & 89 \\
\hline 2 & $2.5654 \mathrm{E}+01$ & $-2.8169 \mathrm{E}-01$ & $1.1318 \mathrm{E}-01$ & $1.5549 \mathrm{E}+00$ & 86 \\
\hline
\end{tabular}

Final oscillation frequency values for the second example (table 2) are $\omega_{R}^{*}=25.6535$; $\omega_{I}^{*}=0.281688$; relative accuracy $\varepsilon_{R}=4.11986 .10^{-8} ; \varepsilon_{I}=5.24144 .10^{-6}$.

The first three lines in the solution protocol are the initial approximations $\omega_{0}, \omega_{0}(1-$ $\varepsilon), \quad \omega_{0}(1-2 \varepsilon)$. The subsequent lines are taken according to Muller.

The example presented in table 2 refers to the most "unsuccessful" one ( 8 iterations; a large difference in frequencies for elastic and viscoelastic problems - 5.4593 and 4.0976). Usually, to find the complex frequency value of structural-inhomogeneous prismatic structures, it is required from 2 to 4 iterations to determine it with a given accuracy.

Table 3.

\begin{tabular}{|c|c|c|c|c|c|c|}
\hline $\begin{array}{l}\text { The number of } \\
\text { waves in the } \\
\text { circumferential } \\
\text { direction. }\end{array}$ & $\begin{array}{c}\text { The number } \\
\text { of waves in } \\
\text { the } \\
\text { longitudinal } \\
\text { direction }\end{array}$ & $\begin{array}{c}\text { Exact } \\
\text { value } \\
{[6]}\end{array}$ & $\begin{array}{c}\text { Ritz } \\
\text { method } \\
{[7]}\end{array}$ & $\begin{array}{c}\text { Reference } \\
\text { paper } \\
{[8]}\end{array}$ & Experiment & $\begin{array}{c}\text { Procedure } \\
\left(\mathbf{M}_{\mathrm{s}}=\mathbf{1 0}\right)\end{array}$ \\
\hline 4 & 1 & 755 & 794 & 796 & 700 & 756 \\
\hline 4 & 2 & 1731 & 1871 & 2086 & 1620 & 1730 \\
\hline 4 & 3 & 2884 & 3091 & 3130 & - & 2886 \\
\hline 5 & 1 & 574 & 595 & 594 & 545 & 573 \\
\hline 5 & 2 & 1272 & 1353 & 1453 & 1210 & 1272 \\
\hline 5 & 3 & 2165 & 2304 & 2332 & - & 2160 \\
\hline 6 & 1 & 533 & 545 & 541 & 525 & 530 \\
\hline 6 & 2 & 1011 & 1059 & 1106 & 980 & 1013 \\
\hline 6 & 3 & 1700 & 1791 & 1812 & 1650 & 1702 \\
\hline
\end{tabular}


2.2. As a test example, the natural oscillations of cylindrical shells of revolution, fixed on the ends, with geometrical parameters $\left(\mathrm{h}=0.25 .10^{-3} \mathrm{~m}, \mathrm{~L}=0.305 \mathrm{~m} ; \mathrm{R}=0.076 \mathrm{~m}\right)$ and physical characteristics $\left(\mathrm{E}=19.6 .10^{10} \mathrm{H} / \mathrm{m}^{2} ;=0.3 ; \rho=7.7 .10^{3} \mathrm{~kg} / \mathrm{m}^{3}\right)$ have been studied. The oscillation frequencies of the shell under consideration (in $\mathrm{Hz}$ ), obtained by a number of authors and by various methods, are given in table 3. A comparison of the calculated frequencies with initial data (table 3 ) leads to good agreement of the results.

2.3. For a thin sphere $\left(\mathrm{E}=1, \rho_{1}=1, \gamma=0.3\right)$ with the ratio of $\mathrm{h} / \mathrm{R}=1 / 100$, a comparative analysis of the lower frequencies $\omega_{R}$ of axisymmetric oscillations and a comparison of the numerical values with the available exact solutions have been given [3]. At $h=1$, the exact solution is $\omega_{1}=0.014359$, the calculated frequency is $\omega_{1}^{p}=0.01440$, the imprecision between the results is about $0.2 \%$.

2.4. The viscoelastic circular truncated conical shell with hinged-supported ends has been calculated. Table 4 shows the dimensionless frequencies $\omega^{*}=\omega L \sqrt{\rho\left(1-\gamma^{2}\right) / E}$, where $\mathrm{L}$ is the length of the shell generatrix. For the half-angle $\alpha=30 ; \gamma=0.3 ; \mathrm{h} / \mathrm{R}_{0}=0.03$; $\mathrm{L}_{1}=0.5 \mathrm{~L}$ (where $\mathrm{R}_{0}$ is the radius of the larger cut of the shell, $\mathrm{L}_{1}$ is the distance along the generatrix from the point of the cone to the smaller cut of the shell) the frequency in a dimensionless form calculated using the proposed method has been compared with the results obtained in [4]. The agreement of the results of numerical implementation with the available data, as in the previous examples, is good.

Table 4.

\begin{tabular}{|c|c|c|c|}
\hline $\begin{array}{c}\text { The number of waves in } \\
\text { the longitudinal direction }\end{array}$ & $\begin{array}{c}\text { The number of waves in } \\
\text { the circumferential } \\
\text { direction }\end{array}$ & $\begin{array}{c}\text { Reference } \\
\text { paper [9] }\end{array}$ & Procedure $\mathbf{M}_{\mathbf{s}}=\mathbf{1 0}$ \\
\hline 1 & 2 & 1.052 & 1.043 \\
\hline 1 & 3 & 0.545 & 0.543 \\
\hline 1 & 4 & 0.530 & 0.5273 \\
\hline 1 & 5 & 0.676 & 0.67 \\
\hline 1 & 6 & 0.812 & 0.811 \\
\hline 1 & 7 & 0.928 & 0.923 \\
\hline 1 & 8 & 1.201 & 1.201 \\
\hline 1 & 9 & 1.488 & 1.485 \\
\hline & 10 & 1.825 & 1.820 \\
\hline
\end{tabular}

3. The accuracy of the construction of oscillation modes can be estimated (similar to constructing the stiffness matrices of shell elements) by symmetry or antisymmetry. As one of the examples, the oscillation modes of a structure whose cross section is shown in figure 3 are given. The computational scheme consists of six cylindrical shells of the same type: each shell element contains 10 orthogonalization points.

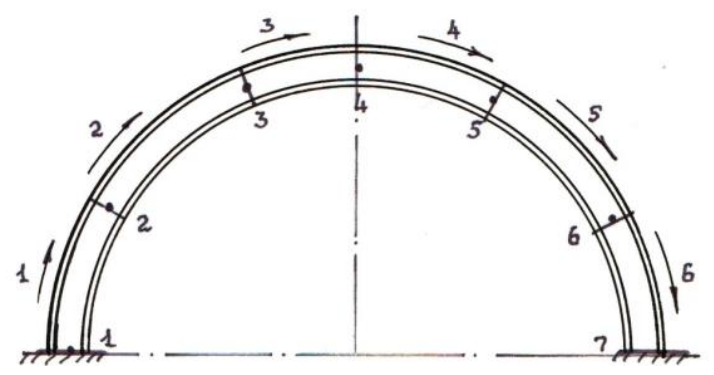

Fig.3. Cross section of a multi-layered cylindrical shell 
The results of determining the oscillations modes of this structure (deflection $w$ ) are given in table 5 .

From this table, the complete antisymmetry of the oscillation modes is seen. Since in this case the deflection in node 4 (the end of the 3rd and the beginning of the 4th shell elements) must be identically equal to zero, its value $w_{4}=9.98 .10^{-6}$ represents a relative error in determining the oscillation mode of the considered structure. Practically, at relative accuracy $\varepsilon=0.001$, when determining the frequencies of natural oscillations, the oscillation mode is determined with an accuracy of up to 4-5 significant digits.

Table 5

\begin{tabular}{|c|c|c|c|c|c|}
\hline $\mathbf{1}$ & $\mathbf{2}$ & $\mathbf{3}$ & $\mathbf{4}$ & $\mathbf{5}$ & $\mathbf{6}$ \\
\hline $0.00 \mathrm{E}+00$ & $1.88 \mathrm{E}-01$ & $-2.25 \mathrm{E}-01$ & $9.98 \mathrm{E}-06$ & $2.25 \mathrm{E}-01$ & $-1.88 \mathrm{E}-01$ \\
\hline$-2.62 \mathrm{E}-02$ & $3.50 \mathrm{E}-01$ & $-4.78 \mathrm{E}-01$ & $2.97 \mathrm{E}-01$ & $-3.23 \mathrm{E}-02$ & $-3.15 \mathrm{E}-02$ \\
\hline$-8.78 \mathrm{E}-02$ & $4.97 \mathrm{E}-01$ & $-7.03 \mathrm{E}-01$ & $5.67 \mathrm{E}-01$ & $-2.69 \mathrm{E}-02$ & $1.02 \mathrm{E}-01$ \\
\hline$-1.61 \mathrm{E}-01$ & $6.10 \mathrm{E}-01$ & $-8.76 \mathrm{E}-01$ & $7.86 \mathrm{E}-01$ & $-4.63 \mathrm{E}-01$ & $1.99 \mathrm{E}-01$ \\
\hline$-2.23 \mathrm{E}-01$ & $6.72 \mathrm{E}-01$ & $-9.79 \mathrm{E}-01$ & $9.34 \mathrm{E}-01$ & $-6.00 \mathrm{E}-01$ & $2.51 \mathrm{E}-01$ \\
\hline$-2.57 \mathrm{E}-01$ & $6.70 \mathrm{E}-01$ & $-1.00 \mathrm{E}-00$ & $1.00 \mathrm{E}-00$ & $-6.70 \mathrm{E}-01$ & $2.57 \mathrm{E}-01$ \\
\hline$-2.51 \mathrm{E}-01$ & $6.00 \mathrm{E}-01$ & $-9.34 \mathrm{E}-01$ & $9.79 \mathrm{E}-01$ & $-6.72 \mathrm{E}-01$ & $2.23 \mathrm{E}-01$ \\
\hline$-1.99 \mathrm{E}-01$ & $6.63 \mathrm{E}-01$ & $-7.86 \mathrm{E}-01$ & $8.76 \mathrm{E}-01$ & $-6.10 \mathrm{E}-01$ & $1.61 \mathrm{E}-01$ \\
\hline$-1.02 \mathrm{E}-01$ & $2.69 \mathrm{E}-01$ & $-5.67 \mathrm{E}-01$ & $7.03 \mathrm{E}-01$ & $-4.97 \mathrm{E}-01$ & $8.78 \mathrm{E}-02$ \\
\hline $3.15 \mathrm{E}-02$ & $3.23 \mathrm{E}-02$ & $-2.97 \mathrm{E}-01$ & $4.78 \mathrm{E}-01$ & $-3.50 \mathrm{E}-01$ & $2.62 \mathrm{E}-02$ \\
\hline $1.88 \mathrm{E}-01$ & $-2.25 \mathrm{E}-01$ & $9.98 \mathrm{E}-06$ & $2.25 \mathrm{E}-01$ & $-1.88 \mathrm{E}-01$ & $-6.61 \mathrm{E}-17$ \\
\hline
\end{tabular}

Comparison with well-known exact analytical solutions (an elastic rectangular plate hinged on the ends, an elastic closed cylindrical shell hinged on the ends) has shown the complete operability of the algorithms. By increasing the number of orthogonalization points (up to 100) and increasing the specified relative accuracy in determining oscillation frequencies (up to $=10^{-10}$ ), it is possible to obtain the solutions with true relative accuracy up to $\varepsilon=10^{-13}$ and with the deviation from the sinusoid, not exceeding $10^{-11}$.

\section{Discussion}

The convergence of the procedure and the proposed algorithm for the case of forced oscillations of shell structures has been tested in 2 stages: the amplitude-frequency characteristics of structure oscillations were built and the resonant frequencies were compared with the frequencies of free oscillations. The resonant frequencies coincided up to the 3rd digit with the corresponding natural frequencies. Further, the solutions of the static problem for a circular plate, cylindrical and conical shells [10] have been compared with the solution by displacements obtained by the proposed procedure at zero frequency of the disturbing force. The relative discrepancy of the results did not exceed $1.2 \%$.

For viscous-elastic structures with a pronounced structural inhomogeneity, the aspect of convergence has been solved as follows. Since the solutions (with a given three-parameter relaxation kernel) or experimental data for the considered multiply-connected structuralinhomogeneous structures could not be found in literature, the correctness of the generalization of the proposed procedure and the developed algorithms for complex arithmetic was tested in the following way. For different values of viscosity, the natural frequencies of a circular plate, cylindrical and conical shells have been determined, their numerical values have been compared with each other and with the solutions of the elastic problem. A similar study was conducted for problems of forced oscillations. As an example, the lower resonant amplitudes of a circular viscous-elastic plate are given 
depending on the viscosity of material. For the relaxation kernel $R(t)=A \exp (-t) t$, the parameter of the kernel A varies. The calculation results are shown in table 6.

Table 6.

\begin{tabular}{|c|c|}
\hline $\begin{array}{c}\text { Parameter A of the kernel R(t) }(\boldsymbol{\alpha}=\mathbf{0 . 1} ; \\
\boldsymbol{\beta}=\mathbf{0 . 0 5})\end{array}$ & Resonant amplitude in the center \\
\hline 0.01 & 40.7 \\
\hline 0.02 & 27.3 \\
\hline 0.03 & 13.9 \\
\hline
\end{tabular}

\section{Conclusions}

Comparative analysis of frequencies and oscillation modes, damping coefficients, resonant frequencies and amplitudes of oscillations of various elements of the considered axisymmetric structural-inhomogeneous shell structures with research results [1-3], [10], [11] allow us to draw the positive conclusions on the convergence and accuracy of developed algorithms in relation to this class of engineering structures; the results can be recommended to be implemented in practice.

\section{References}

1. V.I. Myachenkov, V. Maltsev. Methods and algorithms for calculating spatial structures on a computer (Moscow, Mechanical Engineering, 1984)

2. A.A. Ilyushin, B.E. Pobedrya. Fundamentals of the mathematical theory of thermoviscoelasticity (Moscow, Science, 1970)

3. T. Mavlanov. Strength calculations, Mashinostroenie, 28 (1998)

4. T.M. Mavlanov, N.V. Dremova, G.B. Abdiyeva. Dynamic strength of textile machine elements to the action of a real oscillogram (Tashkent, 2013)

5. L.E. Maltsev. Mechanics of Polymers, 3 (1977)

6. M.M. Mirsaidov. Theory and methods of calculating soil structures for strength and seismic resistance (Tashkent, Fan, 2010)

7. I.A. Birger. Durability, stability, fluctuations Handbook, vol 3 (Moscow, 1968)

8. R.N. Arnold, G.B. Warburton. Proc. Roy. Soc., 197A (1949)

9. V.I. Weingarten. AIAA Journal, 2(4) (1964)

10. L.Yu. Powerus, R.K. Räylit. Tallinn Polytechnic Institute, Series A, 147 (1958)

11. A.A. Maltsev, V.P. Maltsev, V.I. Myachenkov. Mechanics of deformable systems. GGU (1979)

12. M.M. Mirsaidov, T.Z. Sultanov, A. Ishmatov, B. Yuldoshev, E. Toshmatov, D. Magazine of Civil Engineering, 1 (2018)

13. M.M. Mirsaidov, T.Z. Sultonov, B.Yuldoshov, E. Toshmatov, J. Yarashov. J. Irrigation in Land Reclamation, 3 (Tashkent, 2018)

14. Sh. Khudoynazarov, B. Yuldoshov, E. Toshmatov, B. Urinov, J. Yarashov. J. Irrigation in Land Reclamation, 3 (Tashkent, 2018)

15. M.M. Mirsaidov, T.Z. Sultanov, B. Yuldoshev. Irrigation and Melioration (Tashkent, 2018)

16. M.M. Mirsaidov, E. Toshmatov. Irrigation and Melioration (Tashkent, 2018)

17. M.M. Mirsaidov, T.Z. Sultanov, J. Yarashov, Z. Urazmukhamedova. Irrigation and Melioration (Tashkent, 2018)

18. T. Mavlanov, E. Toshmatov, I. Zokirov. Agro Ilm (Tashkent, 2018) 
19. T.Z. Sultanov, J. Yarashov, T. Mavlanov. Agro Ilm (Tashkent, 2018)

20. T.Z. Sultanov, B. Yuldoshev, E. Toshmatov, J. Yarashov, R. Ergashov, M.M. Mirsaidov. MATEC Web Conferences, 265 (2018) 